University of South Carolina

Scholar Commons

8-15-2011

\title{
Dynamics of Spin Relaxation in Finite-Size Two-Dimensional Systems: An Exact Solution
}

\author{
Valeriy A. Slipko \\ Yuriy V. Pershin \\ University of South Carolina - Columbia, pershin@physics.sc.edu
}

Follow this and additional works at: https://scholarcommons.sc.edu/phys_facpub

Part of the Physics Commons

\section{Publication Info}

Published in Physical Review B, ed. Gene D. Sprouse, Volume 84, Issue 7, 2011, pages

075331-1-075331-5.

Slipko, V. A., \& Pershin, Y. V. (2011). Dynamics of spin relaxation in finite-size two-dimensional systems:

An exact solution. Physical Review B, 84(7),075331-1 075331-5.

(c) Physical Review B, 2011, American Physical Society

http://prb.aps.org/abstract/PRB/v84/i7/e075331

This Article is brought to you by the Physics and Astronomy, Department of at Scholar Commons. It has been accepted for inclusion in Faculty Publications by an authorized administrator of Scholar Commons. For more information, please contact digres@mailbox.sc.edu. 


\title{
Dynamics of spin relaxation in finite-size two-dimensional systems: An exact solution
}

\author{
Valeriy A. Slipko ${ }^{1,2}$ and Yuriy V. Pershin ${ }^{1, *}$ \\ ${ }^{1}$ Department of Physics and Astronomy and USC Nanocenter, University of South Carolina, Columbia, South Carolina 29208, USA \\ ${ }^{2}$ Department of Physics and Technology, V. N. Karazin Kharkov National University, Kharkov 61077, Ukraine
}

(Received 15 June 2011; revised manuscript received 21 July 2011; published 16 August 2011)

\begin{abstract}
We find an exact solution for the problem of electron spin relaxation in a two-dimensional (2D) circle with Rashba spin-orbit interaction. Our analysis shows that the spin relaxation in finite-size regions involves three stages and is described by multiple spin relaxation times. It is important that the longest spin relaxation time increases with the decrease in system radius but always remains finite. Therefore, at long times, the spin polarization in small 2D systems decays exponentially with a size-dependent rate. This prediction is supported by results of Monte Carlo simulations.
\end{abstract}

DOI: 10.1103/PhysRevB.84.075331

PACS number(s): 72.15.Lh, 72.25.Dc, 71.70.Ej

\section{INTRODUCTION}

The D'yakonov-Perel ${ }^{1,2}$ spin relaxation mechanism in two-dimensional (2D) systems has attracted wide attention ${ }^{1-14}$ because of its fundamental importance for the field of spintronics. ${ }^{15,16}$ However, the spin relaxation in systems with boundaries is even more important because boundaries are naturally present in all electronic devices. There are only several examples in the literature where the influence of boundary conditions on D'yakonov-Perel' spin relaxation have been explored theoretically and/or experimentally. These examples include investigations of spin relaxation in 2D channels, ${ }^{17-22}$ $2 \mathrm{D}$ half-space, ${ }^{23} 2 \mathrm{D}$ systems with antidots, ${ }^{24}$ large quantum dots, ${ }^{25,26}$ and one-dimensional (1D) finite-length wires. ${ }^{27}$ Both available experimental and theoretical results indicate that, typically in the diffusive spin transport regime, the electron spin life time is longer in systems with boundaries.

In this paper we find an exact solution for the problem of electron spin relaxation in finite-size 2D systems. Specifically, we consider the dynamics of electron spin relaxation in a 2D circle made of a semiconductor structure with Rashba-type spin-orbit interaction. One may think that in small systems the spin relaxation is incomplete as the spin precession angle across the system is small. However, in such a situation, the different effect plays a role: the noncommutativity of spin rotations. Because of this effect, the electron spin precession angle can largely exceed the maximum rotation angle allowed by naive geometrical considerations. To the best of our knowledge, the spin relaxation in small systems was investigated previously only in Ref. 25 . This previous study provides only an asymptotic value of the spin relaxation time without giving details on how the whole process of spin relaxation occurs. In the present paper, we show that the spin relaxation process in finite-size systems is intrinsically complex. The exact solution of this fundamental problem involves an infinite number of spin relaxation constants. At long times, however, only the slowest decaying component survives and the spin polarization exhibits a slow size-dependent exponential decay. Our exact analytical solution is obtained using the Laplace transform ${ }^{28}$ and is confirmed by Monte Carlo simulations of spin dynamics. This work thus provides an important missing part of spin relaxation theory.

This paper is organized as follows. In Sec. II, we introduce a set of diffusive equations describing evolution of spin polarization supplemented by appropriate boundary conditions. The solution of these equations for the case of spin relaxation in a circle is found analytically using the Laplace transform method. The main result of our work is given by Eqs. (22) and (23) describing the time and space dependence of spin polarization components. Section III presents numerical Monte Carlo simulations of spin dynamics that are in an excellent agreement with our analytical results. Section IV summarizes the results and conclusions of the paper.

\section{ANALYTICAL APPROACH}

\section{A. Spin drift-diffusion equations}

Let us consider the dynamics of electron spin polarization in a finite-size 2D electron system such as the circle shown in Fig. 1. The standard electron Hamiltonian with the Rashba spin-orbit interaction term ${ }^{29}$ is given by

$$
\hat{H}=\frac{\hat{\mathbf{p}}^{2}}{2 m}+\alpha(\hat{\sigma} \times \hat{\mathbf{p}}) \cdot \mathbf{z}
$$

where $\hat{\mathbf{p}}=\left(\hat{p}_{x}, \hat{p}_{y}\right)$ is the $2 \mathrm{D}$ electron momentum operator, $m$ is the effective electron's mass, $\hat{\sigma}$ is the Pauli-matrix vector, $\alpha$ is the spin-orbit coupling constant, and $\mathbf{z}$ is a unit vector perpendicular to the confinement plane. The set of diffusion equations $s^{4,30,31}$ for spin polarization can be written as

$$
\begin{aligned}
\frac{1}{D} \frac{\partial \mathbf{S}_{\mathrm{in}}}{\partial t} & =\Delta \mathbf{S}_{\mathrm{in}}+2 \eta \nabla S_{z}-\eta^{2} \mathbf{S}_{\mathrm{in}}, \\
\frac{1}{D} \frac{\partial S_{z}}{\partial t} & =\Delta S_{z}-2 \eta \nabla \cdot \mathbf{S}_{\mathrm{in}}-2 \eta^{2} S_{z},
\end{aligned}
$$

where $\mathbf{S}_{\text {in }}$ and $S_{z}$ are the in-plane and $z$ components of spin polarization, respectively, $D=l^{2} /(2 \tau)$ is the diffusion coefficient, $l$ is the mean free path, $\tau$ is the momentum relaxation time, and $\eta=2 \alpha m \hbar^{-1}$ is the spin rotation angle per unit length (spin rotations are induced by the Rashba spin-orbit interaction). The applicability limits of Eqs. (2) and (3) are $\lambda \ll l \ll a$ and $l \ll \eta^{-1}$, where $\lambda$ is the electron's de Broglie wavelength, and $a$ is the characteristic system size (in the context of this work, $a$ is the radius of the circle). We also note that Eqs. (2) and (3) are valid for any value of $a \eta$. 


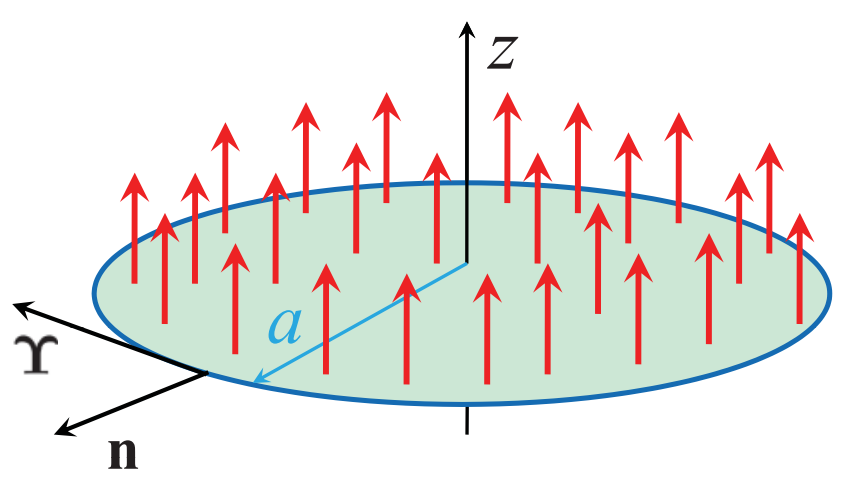

FIG. 1. (Color online) Schematic of a circular 2D region of a radius $a$ with electron spin polarization initially pointing in the $z$ direction perpendicular to the circle plane. $\mathbf{n}$ and $\boldsymbol{\Upsilon}$ are the normal in-plane and tangential vectors to the boundary, correspondingly.

Equations (2) and (3) are supplemented by the standard boundary conditions ${ }^{30,32}$ for spin polarization components

$$
\left(\frac{\partial S_{n}}{\partial n}+\eta S_{z}\right)_{\Gamma}=0, \quad\left(\frac{\partial S_{z}}{\partial n}-\eta S_{n}\right)_{\Gamma}=0, \quad\left(\frac{\partial S_{\Upsilon}}{\partial n}\right)_{\Gamma}=0,
$$

where $\mathbf{n}$ and $\boldsymbol{\Upsilon}$ are the normal in-plane and tangential vectors to the boundary $\Gamma$, respectively. For clarity sake, Fig. 1 illustrates the directions of these vectors at an arbitrary point of the boundary. Physically, the boundary conditions given by Eqs. (4) describe a spin-conserving scattering of electrons from the system boundary. We note that the first two equations of Eqs. (4) are different from the standard zero-flux boundary conditions (e.g., for the heat equation). This is related to the Rashba spin-orbit interaction causing spin rotations. It is clearly seen that the form of the usual von Neumann boundary conditions is restored in the limit of $\eta=0$. We refer to Ref. 30 for more details about boundary conditions in systems with spin-orbit interaction.

Next, we would like to reduce the set of Eqs. (2) and (3) to a single equation for $S_{z}$. Introducing $u=\nabla \cdot \mathbf{S}_{\text {in }}$ and $v=$ $\left(\nabla \times \mathbf{S}_{\text {in }}\right)_{z}$, Eqs. (2) and (3) can be rewritten as

$$
\begin{gathered}
u_{t}=\Delta u+2 \Delta S_{z}-u, \\
\left(S_{z}\right)_{t}=\Delta S_{z}-2 S_{z}-2 u, \\
v_{t}=\Delta v-v .
\end{gathered}
$$

Here, $\Delta$ is the 2D Laplace operator, the time is measured in the units of $t_{s}=\left(D \eta^{2}\right)^{-1}$, and the coordinates are measured in the units of $\eta^{-1}$. Such a convention is used below if not stated otherwise. Combining Eqs. (5) and (6) we readily get

$$
\left(S_{z}\right)_{t t}-2 \Delta\left(S_{z}\right)_{t}+3\left(S_{z}\right)_{t}+\Delta^{2} S_{z}+\Delta S_{z}+2 S_{z}=0 .
$$

\section{B. Analytical solution}

The Laplace transform ${ }^{28}$ of Eq. (8) is given by

$$
\begin{gathered}
\Delta^{2} \tilde{S}_{z}+(1-2 p) \Delta \tilde{S}_{z}+\left(p^{2}+3 p+2\right) \tilde{S}_{z} \\
=\left.(p+1) S_{z}\right|_{t=0}-\left.\Delta S_{z}\right|_{t=0}-\left.2 u\right|_{t=0},
\end{gathered}
$$

where $\tilde{S}_{z}$ is the Laplace transform of $S_{z}$ to the complex $p$ domain. In the above equation, the time derivative of $S_{z}$ at $t=0$ was substituted from Eq. (6).

In what follows we consider the relaxation of homogeneous spin polarization pointing in the $z$ direction perpendicular to the plane of the 2D circle (see Fig. 1). Taking into account the axial symmetry of the problem, the initial and boundary conditions read

$$
\begin{array}{r}
u(r, t=0)=\frac{\partial\left[r S_{r}(r, t=0)\right]}{r \partial r}=0, \quad S_{z}(r, t=0)=S_{0}, \\
\left.\left(\frac{\partial S_{r}}{\partial r}+S_{z}\right)\right|_{r=a}=0,\left.\quad\left(\frac{\partial S_{z}}{\partial r}-S_{r}\right)\right|_{r=a}=0 .
\end{array}
$$

We note that the function $v=\partial\left(r S_{\phi}\right) /(r \partial r)$ is safely taken out of the consideration since the solution $v(r, t)=0$ satisfies Eq. (7) with the boundary condition $\partial S_{\phi} /\left.\partial r\right|_{r=a}=0$ and the initial condition $v(r, 0)=0$. Applying the initial conditions (10), Eq. (9) simplifies to

$$
\begin{aligned}
& \Delta^{2} \tilde{S}_{z}(r)+(1-2 p) \Delta \tilde{S}_{z}(r)+\left(p^{2}+3 p+2\right) \tilde{S}_{z}(r) \\
& \quad=(p+1) S_{0} .
\end{aligned}
$$

The general solution of Eq. (12) can be found by the factorization of its left-hand side and be presented as

$$
\begin{aligned}
\tilde{S}_{z}(r)= & A_{1} J_{0}\left(k_{1} r\right)+A_{2} J_{0}\left(k_{2} r\right)+B_{1} N_{0}\left(k_{1} r\right) \\
& +B_{2} N_{0}\left(k_{2} r\right)+\frac{S_{0}}{p+2},
\end{aligned}
$$

where $A_{1,2}, B_{1,2}$ are arbitrary constants, $J_{0}(x)$ and $N_{0}(x)$ are the zeroth order Bessel and Neumann functions, respectively, and

$$
k_{1,2}^{2}=-p+\frac{1}{2} \pm 2 i \sqrt{p+\frac{7}{16}} .
$$

In the case of the circle, $B_{1,2}=0$ since $N_{0}(x)$ diverges as $x \rightarrow$ 0 . Moreover, the actual choice of two branches corresponding to \pm in Eq. (14) is not essential. We may make a branch cut along the line $p<-7 / 16, \operatorname{Im}(p)=0$ in the plane of complex $p$ and define two branches by the conditions $k_{1,2}^{2}(p=0)=$ $(1 \pm i \sqrt{7}) / 2$.

Although the radial component of spin polarization equals zero at $t=0$, it becomes different than zero at $t>0$ similarly to the case of spin relaxation in rings. ${ }^{33}$ With the help of the Laplace transform of Eq. (6), we express $\tilde{S}_{r}(r)$ through $\tilde{S}_{z}(r)$ as

$$
\begin{aligned}
\tilde{S}_{r}(r)= & \frac{1}{r} \int_{0}^{r} d \xi \xi \tilde{u}(\xi)=\frac{1}{2} \frac{\partial \tilde{S}_{z}(r)}{\partial r}-\frac{p+2}{2 r} \int_{0}^{r} d \xi \xi \tilde{S}_{z}(\xi) \\
& +\frac{1}{2 r} \int_{0}^{r} d \xi \xi S_{z}(\xi, t=0) .
\end{aligned}
$$

The boundary conditions (11) are used to find the values of $A_{1,2}$ in Eq. (13). For this purpose, we Laplace transform Eqs. (11) and employ Eq. (15) to find

$$
\begin{gathered}
\frac{\partial^{2} \tilde{S}_{z}(a)}{\partial r^{2}}+\frac{p+2}{a^{2}} \int_{0}^{a} d r r \tilde{S}_{z}(r)-p \tilde{S}_{z}(a) \\
=\frac{1}{a^{2}} \int_{0}^{a} \operatorname{drr} S_{z}(r, t=0)-S_{z}(a, t=0),
\end{gathered}
$$




$$
\frac{\partial \tilde{S}_{z}(a)}{\partial r}+\frac{p+2}{a} \int_{0}^{a} d r r \tilde{S}_{z}(r)=\frac{1}{a} \int_{0}^{a} d r r S_{z}(r, t=0) .
$$

$A_{1,2}$ are obtained from Eqs. (16) and (17) complemented by Eq. (13) and the initial conditions (10). Finally, the Laplace transform of the $z$ component of the spin polarization is written as

$$
\begin{aligned}
\tilde{S}_{z}(r)= & \frac{2 S_{0}}{(p+2) D(p)}\left[\left(p+2-k_{2}^{2}\right) \frac{J_{1}\left(k_{2} a\right)}{k_{2}} J_{0}\left(k_{1} r\right)\right. \\
& \left.-\left(p+2-k_{1}^{2}\right) \frac{J_{1}\left(k_{1} a\right)}{k_{1}} J_{0}\left(k_{2} r\right)\right]+\frac{S_{0}}{p+2}
\end{aligned}
$$

where the following notation is used:

$$
\begin{aligned}
D(p)= & 2(p+2)\left(k_{2}^{2}-k_{1}^{2}\right) \frac{J_{1}\left(k_{1} a\right) J_{1}\left(k_{2} a\right)}{a k_{1} k_{2}} \\
& +\left[(p+2)\left(k_{1}^{2}-1\right)-p k_{2}^{2}\right] J_{0}\left(k_{1} a\right) \frac{J_{1}\left(k_{2} a\right)}{k_{2}} \\
& -\left[(p+2)\left(k_{2}^{2}-1\right)-p k_{1}^{2}\right] J_{0}\left(k_{2} a\right) \frac{J_{1}\left(k_{1} a\right)}{k_{1}} .
\end{aligned}
$$

The Laplace transform of the $r$ component of spin polarization is found combining Eqs. (15), (18), and (10)

$$
\begin{gathered}
\tilde{S}_{r}(r)=\frac{S_{0}}{(p+2) D(p)}\left[\left(k_{1}^{2}+p+2\right)\left(k_{2}^{2}-p-2\right) \frac{J_{1}\left(k_{2} a\right)}{k_{2}}\right. \\
\left.\quad \times \frac{J_{1}\left(k_{1} r\right)}{k_{1}}-\left(k_{2}^{2}+p+2\right)\left(k_{1}^{2}-p-2\right) \frac{J_{1}\left(k_{1} a\right)}{k_{1}} \frac{J_{1}\left(k_{2} r\right)}{k_{2}}\right] .
\end{gathered}
$$

The inverse Laplace transform of Eqs. (18) and (20) provides the time-domain components of spin polarization. It is important to note that the right-hand sides of Eqs. (18) and (20) do not change under a permutation of $k_{1}$ and $k_{2}$. This means that these functions are one-valued functions in the whole complex plane of $p$ despite the square root in Eq. (14). As a result, $\tilde{S}_{z}(r)$ and $\tilde{S}_{r}(r)$ are meromorphic functions on the whole complex plane of $p$. The poles of these functions are defined by the equation $D(p)=0$, which has an infinite number of roots $p_{n},\left|p_{n}\right| \rightarrow \infty$ as $n \rightarrow \infty$. All poles are characterized by $\operatorname{Im}\left(p_{n}\right)=0$ and $\operatorname{Re}\left(p_{n}\right)<0$. Note that, generally, $p=-2$ is not a pole of both $\tilde{S}_{z}(r)$ and $\tilde{S}_{r}(r)$. Referring to Fig. 2, the positions of the poles depend on the circle radius. At small values of $a \eta$, the values of all $\left|p_{n}\right|$ $(n=1,2, \ldots$,$) increase with the decrease of a \eta$ except for $\left|p_{0}\right|$ whose value decreases. Basically, this is the most interesting pole describing the asymptotic spin relaxation at long times. $p_{0}$ is always located between $-7 / 16$ and 0 and tends to zero when $a \eta \rightarrow 0$, and to $-7 / 16$ when $a \eta \rightarrow \infty$. In the limit of small $a$, an analytical expression for $p_{0}$ can be found. Expanding Eq. (19) over small $p$ and $a$, we get (using dimensional units for $a$ )

$$
-p_{0}=\frac{1}{48}(a \eta)^{4}-\frac{7}{768}(a \eta)^{6}+O\left((a \eta)^{8}\right) .
$$

This expression is basically valid when $a \eta<1$. We also note that the first term in the right-hand side of Eq. (21) was previously reported in Ref. 25. It can also be shown that for $n=1,2, \ldots,\left|p_{n}\right| \sim C_{n}(a \eta)^{-2}$ when $a \eta \ll 1$.

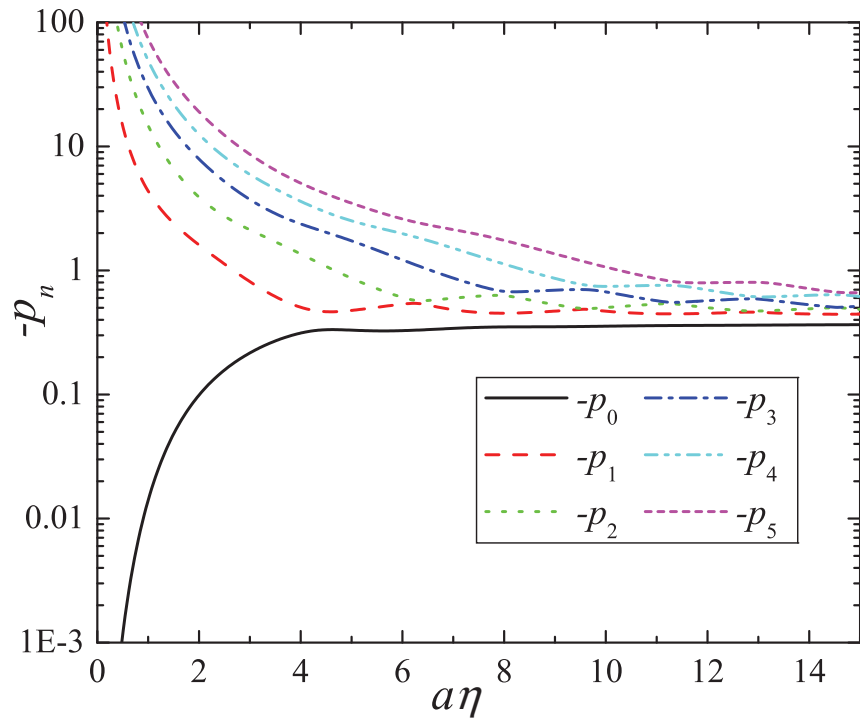

FIG. 2. (Color online) First six poles of $\tilde{S}_{z}(r)$ and $\tilde{S}_{r}(r)$ versus the circle radius. These poles are proportional to the slowest six spin relaxation rates.

The time-domain components of spin polarization are written using the normal dimensional units of time and coordinates as

$$
\begin{gathered}
S_{z}(r, t)=\sum_{n=0}^{+\infty} \frac{2 S_{0} e^{p_{n} D \eta^{2} t}}{\left(p_{n}+2\right) D^{\prime}\left(p_{n}\right)}\left[\left(p_{n}+2-k_{2 n}^{2}\right) \frac{J_{1}\left(k_{2 n} a \eta\right)}{k_{2 n}}\right. \\
\left.\times J_{0}\left(k_{1 n} r \eta\right)-\left(p_{n}+2-k_{1 n}^{2}\right) \frac{J_{1}\left(k_{1 n} a \eta\right)}{k_{1 n}} J_{0}\left(k_{2 n} r \eta\right)\right]
\end{gathered}
$$

and

$$
\begin{aligned}
& S_{r}(r, t)=\sum_{n=0}^{+\infty} \frac{S_{0} e^{p_{n} D \eta^{2} t}}{\left(p_{n}+2\right) D^{\prime}\left(p_{n}\right)}\left[\left(k_{1 n}^{2}+p_{n}+2\right)\right. \\
& \quad \times\left(k_{2 n}^{2}-p_{n}-2\right) \frac{J_{1}\left(k_{2 n} a \eta\right)}{k_{2 n}} \frac{J_{1}\left(k_{1 n} r \eta\right)}{k_{1 n}} \\
& \left.\quad-\left(k_{2 n}^{2}+p_{n}+2\right)\left(k_{1 n}^{2}-p_{n}-2\right) \frac{J_{1}\left(k_{1 n} a \eta\right)}{k_{1 n}} \frac{J_{1}\left(k_{2 n} r \eta\right)}{k_{2 n}}\right] .
\end{aligned}
$$

Equations (22) and (23) represent the main result of this work describing the time dependence of spin relaxation in the circle.

\section{NUMERICAL SIMULATIONS}

To obtain an additional insight on spin relaxation in the 2D circle, we have performed extensive Monte Carlo simulations. All of the specific details of the Monte Carlo simulations approach can be found in Refs. 17 and 34 and will not be repeated here. We just mention that the Monte Carlo simulation program uses a semiclassical description of electron space motion and quantum-mechanical description of spin dynamics. A spin conservation condition was used for electrons elastically scattered from system boundaries. Generally, all obtained Monte Carlo simulation results are in perfect quantitative agreement with our analytical predictions thus confirming 
our analytical theory of spin relaxation in finite-size systems presented in the above section. A comparison of selected analytical and numerical curves is given in Fig. 4.

\section{DISCUSSION AND CONCLUSION}

Basically, the whole process of homogeneous spin relaxation in 2D circles described by Eqs. (22) and (23) can be separated into three main stages. This separation is appropriate for the spin polarization in the central part of the circle at $a \eta \lesssim 3$, when, as it follows from Fig. 2 , the $p_{0}$ pole is well separated from all other poles. The first (initial) stage of spin relaxation takes place at $t \lesssim a^{2} /(16 D)$, where $a^{2} /(4 D)$ is the time it takes for an electron to diffusively propagate over a distance $a$. During this stage, most of the electrons in the circle's center still do not "know about" the presence of the boundary and, therefore, the spin relaxation occurs essentially as in the bulk (according to the standard 2D D'yakonov-Perel' spin relaxation theory). The existence of the first stage of spin relaxation is intuitively clear and well seen in Fig. 4. However, it is difficult to show analytically that Eqs. (22) and (23) reduce to a single exponent at short times since many terms in the sums of Eqs. (22) and (23) play a role during this stage.

In the second stage of spin relaxation, when $a^{2} /(16 D) \lesssim$ $t \lesssim 4 /\left(\left|p_{1}\right| D \eta^{2}\right)$, several exponentially decaying terms play the main role in Eqs. (22) and (23). During this stage, a slow-decaying spin polarization profile is established. In such a profile, $\left|S_{r}\right|$ increases with $r$ as we demonstrate in Fig. 3. Figure 4 shows that at $a \eta=1$ the second stage of spin relaxation is relatively short. For this combination of parameters, the initial fast bulk-type decay of spin polarization (first stage) monotonically transforms into the long-time slow spin polarization decay (third stage). When $a \eta=2$, the second stage of spin relaxation is longer and exhibits a local maxima of $S_{z}$. It is known that the electron spin polarization relaxes slower near sample boundaries than in the bulk. ${ }^{23}$ Therefore, the local maxima of spin polarization can be associated with diffusion

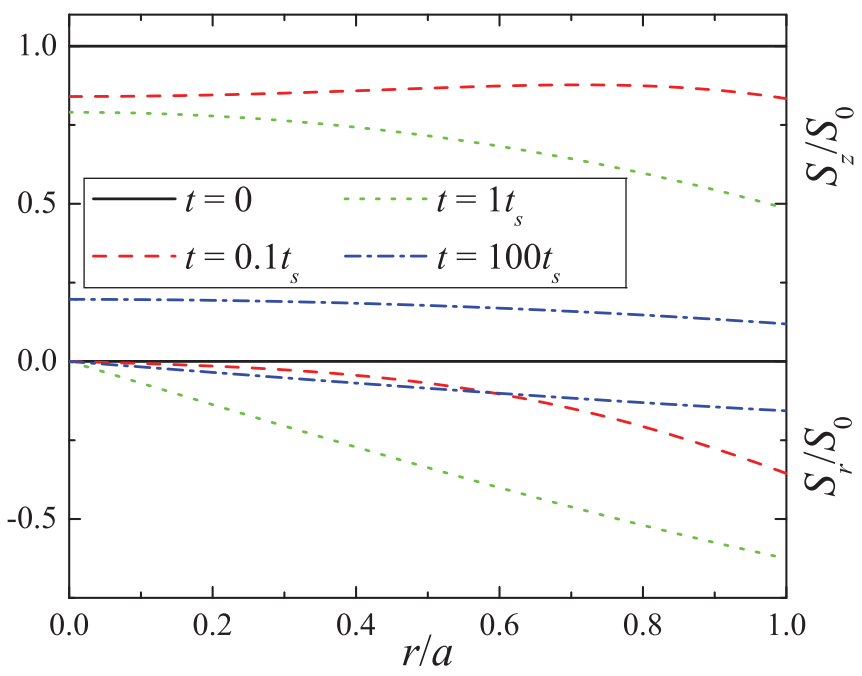

FIG. 3. (Color online) Radial distributions of $S_{r}$ and $S_{z}$ components of spin polarization at different moments of time. Here, the time is measured in units of characteristic bulk spin relaxation time $t_{s}=\left(D \eta^{2}\right)^{-1}$. This plot was obtained at $a \eta=1$.

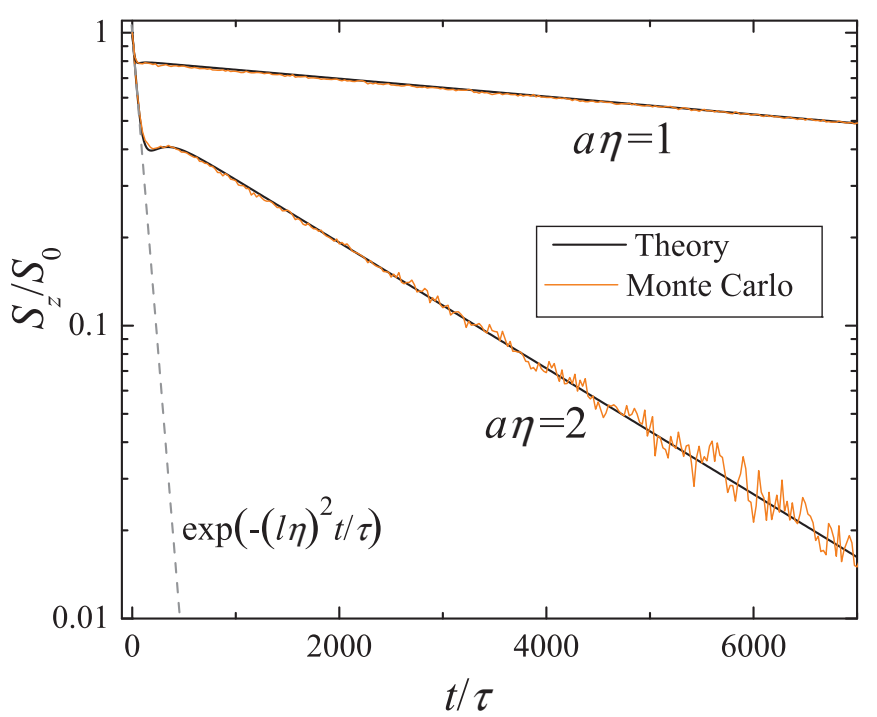

FIG. 4. (Color online) Time dependence of $S_{z}$ component of spin polarization at $r=0$ obtained using Eq. (22) and Monte Carlo calculations. The dashed line corresponds to the usual D'yakonovPerel' relaxation in infinite 2D systems. This plot was obtained using the parameter value $\eta l=0.1$.

of stronger spin polarization from the boundary regions to the circle's center.

The third stage of spin relaxation is a slow single-exponent decay of the spin polarization profile established during the second stage. This process occurs at long times, namely, when $4 /\left(\left|p_{1}\right| D \eta^{2}\right) \lesssim t$. The analytical expressions describing the shape of the slow-decaying spin polarization profile can be easily derived from Eqs. (22) and (23) in the long-time limit when only $p_{0}$ exponential term is important. Explicitly, in the third stage of spin relaxation process, Eqs. (22) and (23) can be reduced to

$$
\begin{aligned}
& S_{z}(r, t)=A(r) e^{-\left|p_{0}\right| D \eta^{2} t}, \\
& S_{r}(r, t)=B(r) e^{-\left|p_{0}\right| D \eta^{2} t} .
\end{aligned}
$$

Here, the functions $A(r)$ and $B(r)$ describe the radial dependence of spin polarization components; their forms can be straightforwardly inferred from Eqs. (22) and (23). The value of $p_{0}$ is determined from Eq. (19). Asymptotically, $p_{0}$ is given by Eq. (21). All three stages of spin relaxation can be easily distinguished in Fig. 4.

In summary, we have found an analytical solution for the problem of electron spin relaxation in the circle in the diffusive electron transport regime. It is shown that a small but nonvanishing spin relaxation exists even in small systems at long times. Consequently, it is not possible to completely eliminate the electron spin relaxation by reducing the system size (remaining in the diffusive transport regime), although the relaxation rate is dramatically suppressed in small-size systems. Basically, the spin relaxation process can be separated into three stages including an initial region of fast bulk-type relaxation, a transition region where the relaxation is described by a combination of several exponentially decaying functions and a region of slow exponential decay at long times. Our results can be easily verified experimentally. 
*pershin@physics.sc.edu

${ }^{1}$ M. I. Dyakonov and V. I. Perel', Fiz. Tverd. Tela (Leningrad) 13, 3581 (1971) [Sov. Phys. Solid State 13, 3023 (1971)].

${ }^{2}$ M. I. Dyakonov and V. Y. Kachorovskii, Sov. Phys. Semicond. 20, 110 (1986).

${ }^{3}$ E. Y. Sherman, Appl. Phys. Lett. 82, 209 (2003).

${ }^{4}$ A. A. Burkov, A. S. Núñez, and A. H. MacDonald, Phys. Rev. B 70, 155308 (2004).

${ }^{5}$ S. Saikin, J. Phys. Condens. Matter 16, 5071 (2004).

${ }^{6}$ Y. V. Pershin, Phys. E 23, 226 (2004).

${ }^{7}$ Y. V. Pershin, Phys. Rev. B 71, 155317 (2005).

${ }^{8}$ I. S. Lyubinskiy and V. Y. Kachorovskii, Phys. Rev. B 73, 041301 (2006).

${ }^{9}$ Y. V. Pershin, Phys. Rev. B 75, 165320 (2007).

${ }^{10}$ M. Q. Weng, M. W. Wu, and H. L. Cui, J. Appl. Phys. 103, 063714 (2008).

${ }^{11}$ P. Kleinert and V. V. Bryksin, Phys. Rev. B 79, 045317 (2009).

${ }^{12}$ Y. V. Pershin and V. A. Slipko, Phys. Rev. B 82, 125325 (2010).

${ }^{13}$ I. V. Tokatly and E. Y. Sherman, Ann. Phys. (NY) 325, 1104 (2010).

${ }^{14}$ I. V. Tokatly and E. Y. Sherman, Phys. Rev. B 82, 161305 (2010).

${ }^{15}$ I. Zutic, J. Fabian, and S. Das Sarma, Rev. Mod. Phys. 76, 323 (2004).

${ }^{16}$ M. Wu, J. Jiang, and M. Weng, Phys. Rep. 439, 61 (2010).

${ }^{17}$ A. A. Kiselev and K. W. Kim, Phys. Rev. B 61, 13115 (2000).

${ }^{18}$ P. Schwab, M. Dzierzawa, C. Gorini, and R. Raimondi, Phys. Rev. B 74, 155316 (2006).
${ }^{19}$ A. W. Holleitner, V. Sih, R. C. Myers, A. C. Gossard, and D. D. Awschalom, New J. Phys. 9, 342 (2007).

${ }^{20}$ C.-H. Chang, J. Tsai, H.-F. Lo, and A. G. Mal'shukov, Phys. Rev. B 79, 125310 (2009).

${ }^{21}$ S. Lüscher, S. M. Frolov, and J. A. Folk, Phys. Rev. B 82, 115304 (2010).

${ }^{22}$ J. Liu, T. Last, E. Koop, S. Denega, B. van Wees, and C. van der Wal, J. Supercond. Nov. Magn. 23, 11 (2010).

${ }^{23}$ Y. V. Pershin, Phys. E 27, 77 (2005).

${ }^{24}$ Y. V. Pershin and V. Privman, Phys. Rev. B 69, 073310 (2004).

${ }^{25}$ I. S. Lyubinskiy, JETP Lett. 83, 336 (2006).

${ }^{26}$ E. J. Koop, B. J. van Wees, D. Reuter, A. D. Wieck, and C. H. van der Wal, Phys. Rev. Lett. 101, 056602 (2008).

${ }^{27}$ V. A. Slipko, I. Savran, and Y. V. Pershin, Phys. Rev. B 83, 193302 (2011).

${ }^{28}$ M. J. Ablowitz and A. S. Fokas, Complex Variables: Introduction and Applications, 2nd ed., (Cambridge University Press, Cambridge, England, 2003).

${ }^{29}$ Y. Bychkov and E. Rashba, JETP Lett. 39, 78 (1984).

${ }^{30}$ V. M. Galitski, A. A. Burkov, and S. Das Sarma, Phys. Rev. B 74, 115331 (2006).

${ }^{31}$ Y. V. Pershin and V. A. Slipko, e-print arXiv:1007.0853v1.

${ }^{32}$ V. A. Slipko and Y. V. Pershin, e-print arXiv:1106.0355v1.

${ }^{33}$ V. A. Slipko and Y. V. Pershin, Europhys. Lett. 95, 37004 (2011).

${ }^{34}$ S. Saikin, Y. V. Pershin, and V. Privman, IEE-Proc. Circ. Dev. Syst. 152, 366 (2005). 